\title{
On One Model of Complex Technical Queuing System with Unreliable Devices and with Time Redundancy
}

\author{
Ramaz R. Shamugia ${ }^{1,2}$ \\ ${ }^{1}$ Research Department of Radiophysics and Electronic Systems Modeling, llia Vekua Sukhumi Institute of \\ Physics and Technology, Tbilisi, Georgia \\ ${ }^{2}$ Faculty of Mathematics and Computer Sciences, Sokhumi State University, Tbilisi, Georgia \\ Email: rmz.shamugia@gmail.com
}

Received 19 January 2014; revised 10 March 2014; accepted 25 April 2014

Copyright ( 2014 by author and Scientific Research Publishing Inc.

This work is licensed under the Creative Commons Attribution International License (CC BY). http://creativecommons.org/licenses/by/4.0/

(c) () Open Access

\section{Abstract}

The given article deals with the development of analytical model of request service process by multichannel technical system with unreliable, repaired and reconfigured service facilities. It is assumed that the system is functioning in service mode of random length random request flows. The system considers the existence of time redundancy for afterservice of calls, the service of which is interrupted with refusal, non-depreciating the performed part of the task. Special probability functions are introduced which on the basis of probability reasoning allow to make the systems of integral equations describing the dynamics of request service process.

\section{Keywords}

QS-Queuing System with Time Backup, Technical Queuing System with Non-Depreciating Refusals, Multichannel QS with Time Backup, QS with Non-Depreciating Refusals, QS with Recovered Devices, QS Networks

\section{Introduction}

Dependability is one of the key problems of contemporary technical systems (TS), also including such systems as hardware and software complexes (HSC), information systems (IS), automated control systems (ACS), diagnostic systems (DS). With the aim to increase dependability the various methods are used concerning the problems of technology, construction, structure, operating rules and service discipline.

One of the most distributed means of dependability increase is introduction of structural redundancy or struc- 
tural (hardware) backup. There exists great enough variety of methods of structural backup, dependability models and their descriptions.

For a long time methods of structural backup were considered universal and with their help it was possible to create high-dependability systems. Though in contemporary conditions when TS represents complex HSC or IS, methods of structural backup in most cases appear not sufficiently effective. Therefore, the attention of designers is more frequently turned to other forms of redundancy: functional, time, informational, algorithmic.

The given work provides the existence of time redundancy-time backup. Time backup represents conditional name of the method of provision of normal functioning of objects performing specified tasks in case of the effect of external disturbances by setting and using of backup (redundancy) time.

Temporary backup is the set of rules of usage and terms of increasing of dependability of technical items leading to origination in an item or its separate devices of redundancy time which may be used for restoration of operability and perfection of technical characteristics.

System failure with time backup (TBS) is the event consisting in such malfunction which leads to the failure of the stated task in given time. This event (system failure) arises in the moment of time reserve exhaustion.

Depending on the consequences TBS elements failures can be non-depreciating (non-destructive) for the performed part of the task, if they cause only delay in task execution and depreciating (destructive) if they make impossible the use of results of the performed work and need repetition of a part or the whole work. In the latter case effective and depreciated lifetime are recognized.

Unlike the other forms of redundancy (structural, functional, informational, loading), backup in the given case is time. This reserve is introduced not in the object, as it happens, for example, at structural backup, but in order (algorithm) of object using (application) as it sometimes happens at informational or functional backup. Classification of the means of time backup can be performed with consideration of the existence of analogy between structural (hardware) and time redundancy. This circumstance enables to distribute, with some stipulation, the existing classification of structural backup on time backup which may be general, separated, group, complete, partial, etc.

In IS time backup can be used for commutation and reconfiguration of the system, for restoration after failures, for repeated execution of works. In HSC especially effective is using of time backup against failures and interferences. It also can be used for increase of the efficiency of other backup forms. Time backup exists in the systems of inertial objects control, where some interruptions in work are allowed. In such systems time backup is determined by inertia of the object and is the about permanent value.

In systems working on request, time backup is a random value. Thus, under the refusal of system with time backup we presume the event after the origination of which the system is no more capable to perform the task at definite conditions of operation. This event (system refusal) arises in the moment of time backup exhaustion.

In works [1]-[9] technical queuing systems with unreliable service devices are considered in different approaches, the results of which, to a large degree, have stipulated further advance in modeling and researching such systems. Nevertheless, the degree of models generalization considered in them, is somewhat lower compared to the one proposed in the given work.

\section{Purpose}

Proceeding from the above said the purpose of the given work is the development of the models of research and probability estimation of the factors of efficiency of functioning of real time failure-proof and high-performance systems having hardware and time backups for the maintenance of the set level of dependability and efficiency and functioning in conditions of the effect of random factors-refusals, repairs and also of random flow of random length tasks for performance and random time of their service.

Further is considered one of the possible and having practical value for organization of service processes, model setting up analytical dependences of these functions on the parameters of the above enumerated different random processes, attending request service.

\section{The Proposed Model Is Developed with Consideration of the Following Assumptions}

Technical queuing system consists of $\mathrm{n}$ identical service facilities; 
Service facilities are subjected to hard failures which obey Poisson law of probability distribution with parameter $\alpha$;

Random process of failed facilities repair is described with exponential law of probability distribution with parameter $\mu$;

Poisson flow of requests incomes into the system for service the intensity of which equals $\lambda$;

Incoming requests are served by exponential law with parameter $\beta$;

Input memory is provided in the system for those requests which enter during time interval, when all $i$ operable devices are busy serving the earlier entered requests;

Queue length is equal to $k-l$ where $k$ is general quantity of requests being in the system in the considered time moment, and $l$ is the quantity of requests served at the given time moment $(l=(0, i))$, where $l$ is the quantity of serving facilities operable by that moment.

Transitions of the system between different states is realized under shared effect of several random processes - processes of incoming and service of demands, as well as processes of refusals and repairs of service facilities:

In this article the model of functioning of technical service system is developed under the following additional assumptions:

The task of constant volume $E=C_{i} u$, where $C_{i}=f(i)$ is total capacity of $i$ operable facilities is performed; Structure and algorithm of executed task allows to divide it into parallel branches in order to distribute between all devices being in operable condition; It is supposed that all facilities composing the system are homogeneous and completely interchangeable and can serve requests in mutual assistance mode.

\section{The Introduced Functions Are Determined as Follows}

$F_{i, j}^{k, k-i}(u)$ is the function of sharing of task performance durations by the system being in state with $i$ operable and $j$ inoperable facilities when in the system there are $k$ requests from which $k-i$ are on service queue and $i$ requests are directly on service.

$G_{i, j}^{k, k-i}(u)$ is the function of sharing of system reconguration time depending on the happening events (refusal or repair of service facilities, income or service of requests);

Refusals happening in the system in the process of functioning are considered as non-depreciating relative to the already performed part of the task.

$H_{i, j}^{(k),(k-i)}(u)=\int_{0}^{u} h_{i, j}^{(k),(k-i)}(v) \mathrm{d} v$ is the probability of the event when service of one of $k$ requests already in service in system with $i$ operable and $j$ inoperable devices in time $u$ will be completed in time less than $u, u+\mathrm{d} u$ by the system staying in the same state $(i, j)$. From the given definition it follows that:

$$
\begin{gathered}
H_{i}^{(k),(k-i)}(u)=\sum_{i=0}^{n} \sum_{j=0}^{n-i} H_{i, j}^{(k),(k-i)}(u), \\
r_{i, j}^{k,(k-i)}(u)=\frac{\mathrm{d}\left[H_{i}^{(k),(k-i)}(u)\right]}{\mathrm{d} u} \frac{1}{1-H_{i}^{(k),(k-i)}(u)}=\frac{h_{i, j}^{(k),(k-u)}(u)}{1-H_{i, j}^{(k),(k-i)}(u)}
\end{gathered}
$$

As it was mentioned above functions $H_{i, j}^{(k),(k-i)}(t)$ describe the process of performing separate tasks. The form of these functions depends on refusal character (non-depreciating or depreciating the already performed part on request service), on control technique (continuous, periodical), on distribution of system reconfiguration time, on afterservice rules (afterservice from interrupted place, repeated performance, etc.).

Further is considered one of the possible and having practical value for organization of service processes, model setting up analytical dependences of these functions on the parameters of the above enumerated different random processes, attending request service.

In the given article the model of functioning of technical service system is developed under the following assumptions:

- The task of constant volume $E=C_{i} u$, where $C_{i}=f(i)$ is total capacity of $i$ operable facilities is performed;

- Structure and algorithm of executed task allows to divide it into parallel branches in order to distribute between all devices being in operable condition;

- It is supposed that all facilities composing the system are homogeneous and completely interchangeable and can serve requests in mutual assistance mode;

- $F_{i, j}^{k, k-i}(u)$ is the function of sharing of task performance durations by the system being in state with $i$ oper- 
able and $j$ inoperable facilities when in the system there are $k$ requests from which $k-i$ are on service queue and $i$ requests are directly on service.

- $G_{i, j}^{k, k-i}(u)$ is the function of sharing of system reconfiguration time depending on the happening events (refusal or repair of service facilities, income or service of requests);

- Refusals happening in the system in the process of functioning are considered as non-depreciating relative to the already performed part of the task.

In order to describe the process of system functioning in the above presented statement, introduce function $H_{i, j}^{(k)(k-i)}\left(t, \tau_{z}\right)$ the probability of the event when task performance with length $\tau_{z}$ (minimum time of task performance at operable condition of all $n$ facilities) will be completed for time less than $t$, by the system being in state with $i$ operable and $j$ inoperable facilities, in condition that at the beginning of task service the system was in one of the possible states $(i, j), i=0, n ; j=n-i$.

$$
\begin{aligned}
H_{i, j}^{(k)(k-i)}\left(t, \tau_{z}\right)= & \int_{0}^{t} \mathrm{e}^{-[(i \alpha+j \mu+(k-i) \lambda+i \beta]} u \mathrm{~d} F_{i, j}^{k, k-i}\left(C_{i} u\right) H_{i, j}^{k, k-i}(t-u) \\
& +i \alpha \int_{0}^{t} \mathrm{e}^{-[(i \alpha+j \mu+(k-l) \lambda+i \beta] u}\left[1-F_{i, j}^{k, k-i}\left(C_{i} u\right)\right] \mathrm{d} u \int_{0}^{t-u} \mathrm{~d} G_{i-1, j+1}^{k, k-i}(v) H_{i-1, j+1}^{k, k-i}\left(t-u-v, \tau_{z}-C_{i} u\right) \\
& +j \mu \int_{0}^{t} \mathrm{e}^{-[(i \alpha+j \mu+(k-i) \lambda+i \beta] u}\left[1-F_{i, j}^{k, k-i}\left(C_{i} u\right)\right] \mathrm{d} u \int_{0}^{t-u} \mathrm{~d} G_{i+1, j-1}^{k, k-i}(v) H_{i+1, j-1}^{k, k-i}\left(t-u-v, \tau_{z}-C_{i} u\right) \\
& +(k-i) \lambda \int_{0}^{t} \mathrm{e}^{-[(i \alpha+j \mu+(k-i) \lambda+i \beta] u}\left[1-F_{i, j}^{k+1, k-i}\left(C_{i} u\right)\right] \mathrm{d} u \int_{0}^{t-u} \mathrm{~d} G_{i, j}^{k, k+1-i}(v) H_{i, j}^{k, k+1-i}\left(t-u-v, \tau_{z}-C_{i} u\right) \\
& +i \beta \int_{0}^{t} \mathrm{e}^{-[(i \alpha+j \mu+(k-i) \lambda+i \beta] u}\left[1-F_{i, j}^{k, k-i}\left(C_{i} u\right)\right] \mathrm{d} u \int_{0}^{t-u} \mathrm{~d} G_{i, j}^{k, k-1-i}(v) H_{i, j}^{k, k-1-i}\left(t-u-v, \tau_{z}-C_{i} u\right) ;
\end{aligned}
$$

Explain probability meaning of summands in this integral equation:

- $\int_{0}^{t} \mathrm{e}^{-[(i \alpha+j \mu+(k-i) \lambda+i \beta] u} \mathrm{d} F_{i, j}^{k, k-i}\left(C_{i} u\right) H_{i, j}^{k, k-i}(t-u)$ is the probability of joint event when:

1) During time $u$ in time interval $(0, t)$ will happen neither of the following events: refusal of no one of $i$ operable facilities, repair of no one of $j$ facilities being in refusal state, income for service of no one of $k-i$ requests being in queue, service of no one of $i$ requests being on service.

- $\quad i \alpha \int_{0}^{t} \mathrm{e}^{-[(i \alpha+j \mu+(k-l) \lambda+i \beta] u}\left[1-F_{i-1, j+1}^{k, k-i}\left(C_{i} u\right)\right] \mathrm{d} u \int_{0}^{t-u} \mathrm{~d} G_{i-1, j+1}^{k, k-i}(v) H_{i-1, j+1}^{k, k-i}\left(t-u-v, \tau_{z}-C_{i} u\right)$ is the probability of joint event when:

1) $i \alpha \mathrm{e}^{-[(i \alpha+j \mu+(k-l) \lambda+i \beta] u}$-in moment $u$ in time interval $(0, t)$ will happen refusal of one of $i$ facilities being in operable states;

2) $\left[1-F_{i, j}^{k, k-i}\left(C_{i} u\right)\right] \mathrm{d} u$ - during time interval $u$ will be performed a part of task equal to $\left(C_{i} u\right)$, but it will not be fully completed;

3) $\mathrm{d} G_{i-1, j+1}^{k, k-i}(v)$-in moment $v$ in time interval $(0, t-u)$ will happen reconfiguration of the system;

4) $H_{i-1, j+1}^{k, k-i}\left(t-u-v, \tau_{z}-C_{i} u\right)$ in time interval $(t-u-v)$ will happen afterservice of the remaining part of task $\tau_{z}-C_{i} u$ by the system being in state $H_{i-1, j+1}^{k, k-i}(t-u-v)$;

- $j \mu \int_{0}^{t} \mathrm{e}^{-[(i \alpha+j \mu+(k-i) \lambda+i \beta] u}\left[1-F_{i+1, j-1}^{k, k-i}\left(C_{i} u\right)\right] \mathrm{d} u \int_{0}^{t-u} \mathrm{~d} G_{i+1, j-1}^{k, k-i}(v) H_{i+1, j-1}^{k, k-i}\left(t-u-v, \tau_{z}-C_{i} u\right)$ is the probability of joint event when:

1) $j \mu \mathrm{e}^{-[(i \alpha+j \mu+(k-i) \lambda+i \beta] u}$-in moment $u$ in time interval $(0, t)$ will happen repair of one of $j$ facilities being in refusal state;

2) $\left[1-F_{i, j}^{k, k-i}\left(C_{i} u\right)\right] \mathrm{d} u$-during time interval $u$ will be performed a part of task equal to $\left(C_{i} u\right)$, but it will not be fully completed;

3) $\mathrm{d} G_{i+1, j-1}^{k, k-i}(v)$-in moment $v$ in time interval $(0, t-u)$ will happen reconfiguration of the system;

4) $H_{i+1, j-1}^{k, k-i}\left(t-u-v, \tau_{z}-C_{i} u\right)$-in time interval $(t-u-v)$ will happen afterservice of the remaining part of event $\tau_{z}-C_{i} u$ by the system being in state $H_{i+1, j-1}^{k, k-i}(t-u-v)$;

- $(k-i) \lambda \int_{0}^{t} \mathrm{e}^{-[(i \alpha+j \mu+(k-1) \lambda+i \beta] u}\left[1-F_{i, j}^{k+1, k+1-i}\left(C_{i} u\right)\right] \mathrm{d} u \int_{0}^{t-u} \mathrm{~d} G_{i, j}^{k, k+1-i}(v) H_{i, j}^{k, k+1-i}\left(t-u-v, \tau_{z}-C_{i} u\right)$ is the proba- 
bility of joint event when:

1) $(k-i) \lambda \mathrm{e}^{-[(i \alpha+j \mu+(k-i) \lambda+i \beta] u}$-in moment $\mathrm{u}$ in time interval $(0, t)$ will happen income of one of $(k-i)$ calls being on service in call stacker;

2) $\left[1-F_{i, j}^{k, k-i}\left(C_{i} u\right)\right] \mathrm{d} u$-during time interval u will be performed a part of task equal to $\left(C_{i} u\right)$, but it will not be fully completed;

3) $\mathrm{d} G_{i, j}^{k, k+1-i}(v)$-before moment $v$ in time interval $(0, t-u)$ will happen reconfiguration of the system;

4) $H_{i, j}^{k, k+1-i}\left(t-u-v, \tau_{z}-C_{i} u\right)$-in time interval $(t-u-v)$ will happen afterservice of the remained part of task $\tau_{z}-C_{i} u$ by the system being in state $H_{i, j}^{k, k+1-i}(t-u-v)$.

- $\quad i \beta \int_{0}^{t} \mathrm{e}^{-[(i \alpha+j \mu+(k-i) \lambda+i \beta] u}\left[1-F_{i, j}^{k, k-i}\left(C_{i} u\right)\right] \mathrm{d} u \int_{0}^{t-u} \mathrm{~d} G_{i, j}^{k, k-1-i}(v) H_{i, j}^{k, k-1-i}\left(t-u-v, \tau_{z}-C_{i} u\right)$ is the probability of joint event when:

1) $i \beta \mathrm{e}^{-[(i \alpha+j \mu+(k-i) \lambda+i \beta] u}$-in moment $u$ in time interval $(0, t)$ will happen serving of one of $i$ requests being on service;

2) $\left[1-F_{i, j}^{k, k-i}\left(C_{i} u\right)\right] \mathrm{d} u$-during time interval $u$ will be performed a part of task equal to $\left(C_{i} u\right)$, but it will not be fully completed.

3) $\mathrm{d} G_{i, j}^{k, k-1-i}(v)$ - up to moment $v$ in time interval $(0, t-u)$ will happen reconfiguration of the system;

4) $H_{i, j}^{k, k-1-i}\left(t-u-v, \tau_{z}-C_{i} u\right)$-in time interval $(t-u-v)$ will happen afterservice of the remained part of task $\tau_{z}-C_{i} u$ by the system being in state $H_{i, j}^{k, k-1-i}(t-u-v)$.

$$
\begin{gathered}
L\left\{H_{i, j}^{k, k-i}\left(t, \tau_{z}\right)\right\}=\overline{\bar{H}}_{i, j}^{k, k-i}(s, \omega)=\int_{0}^{\infty} \mathrm{e}^{-\omega \tau_{z}}\left[\int_{0}^{\infty} \mathrm{e}^{-s t} H_{i, j}^{k, k-i}\left(t, \tau_{z}\right) \mathrm{d} t\right] \\
L\left\{F_{i, j}^{k, k-i}\left(C_{i} u\right)\right\}=\frac{1}{C_{i}} \bar{F}_{i, j}^{k, k-1}\left(s / C_{i}\right)=(1 / s) \mathrm{e}^{-\tau_{0}\left(s / C_{i}\right)} \\
L\left\{G_{i, j}^{k, k-i}(v)\right\}=\bar{G}_{i, j}^{k, k-i}(s)=\int_{0}^{\infty} \mathrm{e}^{-s t} G_{i, j}^{k, k-i}(v) \mathrm{d} v
\end{gathered}
$$

Applying Laplace transformation by both arguments $t$ and $\tau$ to Equation (3) for separate summands in this equation we receive:

- $\int_{0}^{t} \mathrm{e}^{-[(i \alpha+j \mu+(k-i) \lambda+i \beta] u} \mathrm{d} F_{i, j}^{k, k-i}\left(C_{i} u\right) H_{i, j}^{k, k-i}(t-u)=\left[(1 / s) \mathrm{e}^{-\tau_{\tau}[s+i \alpha+j \mu+(k-i) \lambda+i \beta]} / C_{i}\right] * \bar{H}_{i, j}^{k, k-i}(s)$

$$
L\left\{i \alpha \int_{0}^{t} \mathrm{e}^{-[(i \alpha+j \mu+(k-l) \lambda+i \beta] u}\left[1-F_{i-1, j+1}^{k, k-i}\left(C_{i} u\right)\right] \mathrm{d} u \int_{0}^{t-u} \mathrm{~d} G_{i-1, j+1}^{k, k-i}(v) H_{i-1, j+1}^{k, k-i}\left(t-u-v, \tau_{z}-C_{i} u\right)\right\}
$$

- $=\frac{i \alpha\left[C_{i}-\mathrm{e}^{\tau_{z}[s+i \alpha+j \mu+(k-i) \lambda+i \beta]}\right]}{C_{i}\{[s+i \alpha+j \mu+(k-i) \lambda+i \beta]+\omega\}} \bar{G}_{i-1, j+1}^{k, k-i}(s) \overline{\bar{H}}_{i-1, j+1}^{k, k-i}(s, \omega)$;

$$
L\left\{j \mu \int_{0}^{t} \mathrm{e}^{-[(i \alpha+j \mu+(k-i) \lambda+i \beta] u}\left[1-F_{i+1, j-1}^{k, k-i}\left(C_{i} u\right)\right] \mathrm{d} u \int_{0}^{t-u} \mathrm{~d} G_{i+1, j-1}^{k, k-i}(v) H_{i+1, j-1}^{k, k-i}\left(t-u-v, \tau_{z}-C_{i} u\right)\right\}
$$

- $=\frac{j \mu\left[C_{i}-\mathrm{e}^{\tau_{z}[s+i \alpha+j \mu+(k-i) \lambda+i \beta]}\right]}{C_{i}\{[s+i \alpha+j \mu+(k-i) \lambda+i \beta]+\omega\}} \bar{G}_{i+1, j-1}^{k, k-1}(s) \overline{\bar{H}}_{i+1, j-1}^{k, k-1}(s, \omega)$;

$$
L\left\{(k-i) \lambda \int_{0}^{t} \mathrm{e}^{-[(i \alpha+j \mu+(k-i) \lambda+l \beta] u}\left[1-F_{i, j}^{k+1, k+1-i}\left(C_{i} u\right)\right] \mathrm{d} u \int_{0}^{t-u} \mathrm{~d} G_{i, j}^{k, k+1-i}(v) H_{i, j}^{k, k+1-i}\left(t-u-v, \tau_{z}-C_{i} u\right)\right\}
$$

- $=\frac{(k-i) \lambda\left[C_{i}-\mathrm{e}^{\tau_{z}[s+i \alpha+j \mu+(k-i) \lambda+i \beta]}\right]}{C_{i}\{[s+i \alpha+j \mu+(k-i) \lambda+i \beta]+\omega\}} \bar{G}_{i, j}^{k, k+1-i}(s) \overline{\bar{H}}_{i, j}^{k, k+1-i}(s, \omega)$; 


$$
\begin{aligned}
& L\left\{i \beta \int_{0}^{t} \mathrm{e}^{-[(i \alpha+j \mu+(k-i) \lambda+l \beta] u}\left[1-F_{i, j}^{k, k-i}\left(C_{i} u\right)\right] \mathrm{d} u \int_{0}^{t-u} \mathrm{~d} G_{i, j}^{k, k-1-i}(v) H_{i, j}^{k, k-1-i}\left(t-u-v, \tau_{z}-C_{i} u\right)\right\} \\
& =\frac{i \beta\left[C_{i}-\mathrm{e}^{\tau_{z}[s+i \alpha+j \mu+(k-i) \lambda+i \beta]}\right]}{C_{i}\{[s+i \alpha+j \mu+(k-i) \lambda+i \beta]+\omega\}} \bar{G}^{k, k-1-i}(s) \overline{\bar{H}}_{i, j}^{k, k-1-i}(s, \omega) .
\end{aligned}
$$

Substituting the received expressions of Laplace transformations in Equation (3) we receive the following system of algebraic equations:

$$
\begin{aligned}
\overline{\bar{H}}_{i, j}^{k, k-i}(s, \omega)= & {\left[(1 / s) \mathrm{e}^{-\tau_{z}[s+i \alpha+j \mu+(k-i) \lambda+i \beta]} / C_{i}\right] * \overline{\bar{H}}_{i, j}^{k, k-i}(s, \omega) } \\
& +\frac{i \alpha\left[C_{i}-\mathrm{e}^{\tau_{z}[s+i \alpha+j \mu+(k-i) \lambda+i \beta]}\right]}{C_{i}\{[s+i \alpha+j \mu+(k-i) \lambda+i \beta]+\omega\}} \bar{G}_{i-1, j+1}^{k, k-i}(s) \overline{\bar{H}}_{i-1, j+1}^{k, k-1}(s, \omega) \\
& +\frac{j \mu\left[C_{i}-\mathrm{e}^{\tau_{z}[s+i \alpha+j \mu+(k-i) \lambda+i \beta]}\right]}{C_{i}\{[s+i \alpha+j \mu+(k-i) \lambda+i \beta]+\omega\}} \bar{G}_{i+1, j-1}^{k, k-1}(s) \overline{\bar{H}}_{i+1, j-1}^{k, k-1}(s, \omega) \\
& +\frac{(k-i) \lambda\left[C_{i}-\mathrm{e}^{\tau_{z}[s+i \alpha+j \mu+(k-i) \lambda+i \beta]}\right]}{C_{i}\{[s+i \alpha+j \mu+(k-i) \lambda+i \beta]+\omega\}} \bar{G}_{i, j}^{k, k+1-i}(s) \overline{\bar{H}}_{i, j}^{k, k+1-i}(s, \omega) \\
& +\frac{i \beta\left[C_{i}-\mathrm{e}^{\tau_{z}[s+i \alpha+j \mu+(k-i) \lambda+i \beta]}\right]}{C_{i}\{[s+i \alpha+j \mu+(k-i) \lambda+i \beta]+\omega\}} \bar{G}^{k, k-1-i}(s) \overline{\bar{H}}_{i, j}^{k, k-1-i}(s, \omega)
\end{aligned}
$$

Introducing denotation $\Psi(i, j, k, \mu)=\frac{\left[C_{i}-\mathrm{e}^{\tau_{z}[s+i \alpha+j \mu+(k-i) \lambda+i \beta]}\right]}{C_{i}\{[s+i \alpha+j \mu+(k-i) \lambda+i \beta]+\omega\}}$

the system of equations can be transformed to the following form:

$$
\begin{aligned}
& \left\{1-\Psi(i, j, k, \mu)\left[(1 / s) \mathrm{e}^{-\tau_{\tau}[s+i \alpha+j \mu+(k-i) \lambda+i \beta]} / C_{i}\right]\right\} * \overline{\bar{H}}_{i, j}^{k, k-i}(s, \omega) \\
& =i \alpha \bar{G}_{i-1, j+1}^{k, k-1}(s) \overline{\bar{H}}_{i-1, j+1}^{k, k-1}(s, \omega)+j \mu \bar{G}_{i+1, j-1}^{k, k-1}(s) \overline{\bar{H}}_{i+1, j-1}^{k, k-1}(s, \omega) \\
& +(k-i) \lambda \bar{G}_{i, j}^{k, k+1-i}(s) \overline{\bar{H}}_{i, j}^{k, k+1-i}(s, \omega)+i \beta \bar{G}_{i, j}^{k, k-1-i}(s) \overline{\bar{H}}_{i, j}^{k, k-1-i}(s, \omega) ;
\end{aligned}
$$

The given system of equations is split into two subsystems depending on which group of indices $\overline{\bar{H}}_{i, k}^{k, k-i}(s, \omega)$ is considered fixed, upper $(k, k-i)$ or lower $(i, j)$.

1) At fixed upper indices $(k-i) \lambda=0 ; i \beta=0$. Thus:

$$
(k-i) \lambda \bar{G}_{i, j}^{k, k+1-i}(s) \overline{\bar{H}}_{i, j}^{k, k+1-i}(s, \omega)=0 ; \quad i \beta \bar{G}_{i, j}^{k, k-1-i}(s) \overline{\bar{H}}_{i, j}^{k, k-1-i}(s, \omega)=0
$$

and relative to $\overline{\bar{H}}_{i, j}^{k, k-i}(s, \omega)$ we receive difference subsystem of algebraic equations:

$$
\begin{aligned}
& i \alpha \bar{G}_{i-1, j+1}^{k, k-i}(s) \overline{\bar{H}}_{i-1, j+1}^{k, k-i}(s, \omega)+\left\{1-\Psi(i, j, k, \mu)\left[(1 / s) \mathrm{e}^{-\tau_{z}[s+i \alpha+j \mu]} / C_{i}\right]\right\} * \overline{\bar{H}}_{i, j}^{k, k-i}(s, \omega) \\
& +j \mu \bar{G}_{i+1, j-1}^{k, k-i}(s) \overline{\bar{H}}_{i+1, j-1}^{k, k-i}(s, \omega)=0 ; \quad \text { for } i \neq 0, j \neq 0
\end{aligned}
$$

Here: $\Psi(i, j, k, \mu)=\frac{\left[C_{i}-\mathrm{e}^{\tau_{z}[s+i \alpha+j \mu]}\right]}{C_{i}\{[s+i \alpha+j \mu]+\omega\}}$.

2) At fixed lower indices $i \alpha=0 ; j \mu=0$; Thus:

$$
j \mu \bar{G}_{i+1, j-1}^{k, k-1}(s) \overline{\bar{H}}_{i+1, j-1}^{k, k-1}(s, \omega)=0 ; \quad i \alpha \bar{G}_{i-1, j+1}^{k, k-i}(s) \overline{\bar{H}}_{i-1, j+1}^{k, k-i}(s, \omega)=0
$$

and relative to $\overline{\bar{H}}_{i, j}^{k, k-i}(s, \omega)$ we receive difference subsystem of algebraic equations: 


$$
\begin{aligned}
& i \beta \bar{G}_{i, j}^{k, k-1-i}(s) \overline{\bar{H}}_{i, j}^{k, k-1-i}(s, \omega)+\left\{1-\Psi(i, j, k, \mu)\left[(1 / s) \mathrm{e}^{-\tau_{\tau}[s+(k-i) \lambda+i \beta]} / C_{i}\right]\right\} * \overline{\bar{H}}_{i, j}^{k, k-i}(s, \omega) \\
& +(k-i) \lambda \bar{G}_{i, j}^{k, k+1-i}(s) \overline{\bar{H}}_{i, j}^{k, k+1-i}(s, \omega)=0 ; \quad \text { for } i \neq 0, j \neq 0
\end{aligned}
$$

Here: $\Psi(i, j, k, \mu)=\frac{\left[C_{i}-\mathrm{e}^{\tau_{z}[s+(k-i) \lambda+i \beta]}\right]}{C_{i}\{[s+(k-i) \lambda+i \beta]+\omega\}}$.

After simple transformations equation systems (6) and (7) are reduced to the form which is easily solved relative to $\overline{\bar{H}}_{i, j}^{k, k-i}(s, \omega)$ when $i \neq 0, j \neq 0$ with the help of Cramer methods.

As an example we give construction of main determinant of equations system (6) denoting it by $\overline{\bar{\Delta}}_{0}(s, \omega)$. It will consist of coefficients in case of unknown functions $\overline{\bar{H}}_{i-1, j+1}^{k, k-i}(s, \omega), \overline{\bar{H}}_{i, j}^{k, k-i}(s, \omega), \overline{\bar{H}}_{i+1, j-1}^{k, k-i}(s, \omega)$ for different values of $(i, j)$, where $i=1, n ; j=1, n-i$.

$$
\begin{aligned}
& \overline{\bar{H}}_{i-1, j+1}^{k, k-i}(s, \omega) \quad \overline{\bar{H}}_{i, j}^{k, k-i}(s, \omega) \quad \overline{\bar{H}}_{i+1, j-1}^{k, k-i}(s, \omega)
\end{aligned}
$$

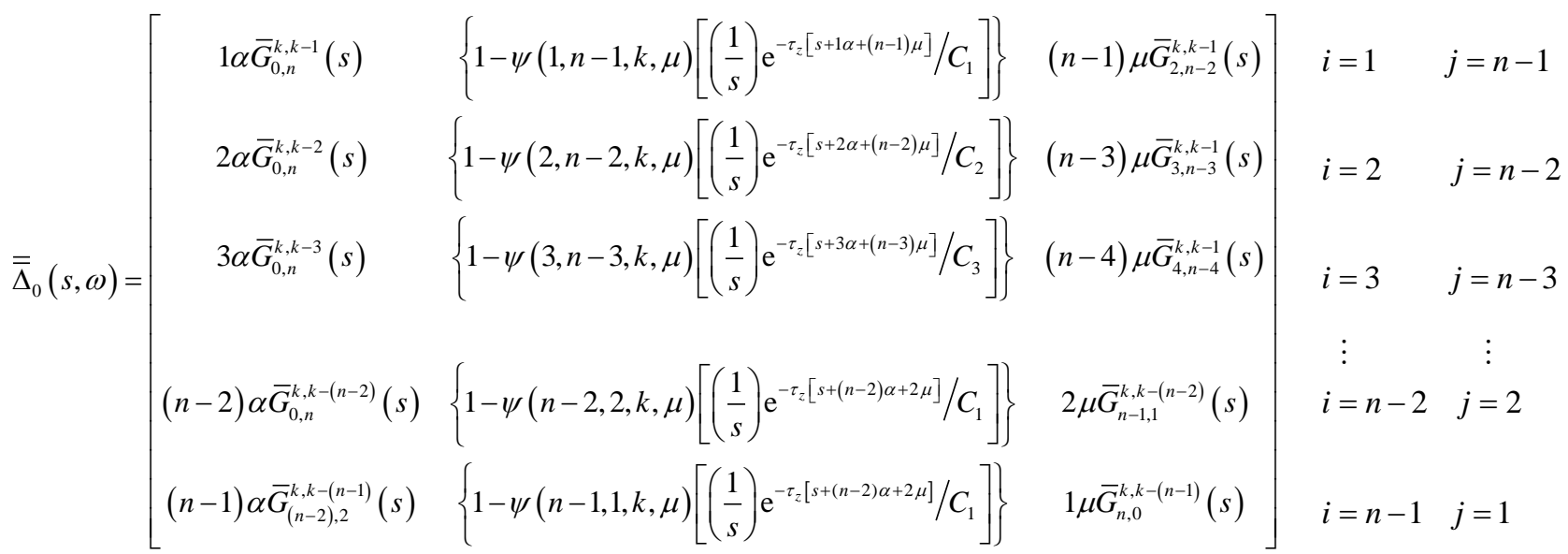

Designating by $\overline{\bar{\Delta}}_{i-1, j+1}^{k, k-i}(s, \omega), \quad \overline{\bar{\Delta}}_{i, j}^{k, k-i}(s, \omega)$ and $\overline{\bar{\Delta}}_{i+1, j-1}^{k, k-i}(s, \omega)$ the determinants received by substitution of the corresponding columns of main determinants by zeroes, the solution of Equation (6) can be written as follows:

$$
\begin{aligned}
& \overline{\bar{H}}_{i-1, j+1}^{k, k-i}(s, \omega)=\overline{\bar{\Delta}}_{i-1, j+1}^{k, k-i}(s, \omega) / \overline{\bar{\Delta}}_{0}(s, \omega) ; \\
& \overline{\bar{H}}_{i, j}^{k, k-i}(s, \omega)=\overline{\bar{\Delta}}_{i, j}^{k, k-i}(s, \omega) / \overline{\bar{\Delta}}_{0}(s, \omega) ; \\
& \overline{\bar{H}}_{i+1, j-1}^{k, k-i}(s, \omega)=\overline{\bar{\Delta}}_{i+1, j-1}^{k, k-i}(s, \omega) / \overline{\bar{\Delta}}_{0}(s, \omega) .
\end{aligned}
$$

Similarly is solved the system of Equation (7) with consideration that in this case the values of lower indices $(i, j)$ are fixed and functions $\overline{\bar{H}}_{i, j}^{k, k-1-i}(s, \omega), \overline{\bar{H}}_{i, j}^{k, k-i}(s, \omega)$ and $\overline{\bar{H}}_{i, j}^{k, k+1-i}(s, \omega)$ are target ones.

For determination of function $\overline{\bar{H}}_{i, j}^{k, k-i}(s, \omega)$ for case $i=0, j=0$ it is essential that in Equations (6) and (7) be assumed by turns that $i=0, j=0, k-i=0$. At the same time the corresponding summands in Equations (6) and (7) turn into zero and after simple transformations we get systems of algebraic equations for determination of the mentioned functions.

\section{Conclusion}

The presented work gives the development of analytical model of multichannel technical queuing system with time redundancy. Non-depreciating character of refusals, the presence of the systems of repair of failed serving facilities and of reconfiguration of the system after refusals and repair are assumed. As to random processes determining system state, minimum limitations are admitted. The model provides derivation of the function for distribution of task performing time which will allow estimate probability states of the described system on design as well as operation stage. The model is the most generalized as compared to those known by literature. 


\section{References}

[1] Cherkesov, G.N. (2005) Durability of Software-Hardware Complexes. Piter, 479.

[2] Polovko, A.M. and Gurov, C.V. (2008) Fundamentals of the Theory of Durability. Petergurg, 704.

[3] Gnedenko, B.V. and Kovalenko, I.N. (2012) Introduction to Queuing Theory. LKT, 400.

[4] Saati, T.L. (1965) Elements of Queuing Theory and Its Application. Sovetskoe Radio, 510.

[5] Mikadze, Z.I., Mikadze, I.S. and Khocholava, V.V. (2007) On One Multichannel Mixed Queuing System with Limited Waiting Time. Automatics and Telemechanics, 7, 44-51.

[6] Khurodze, R.A., Khocholava, V.V. and Mikadze, I.S. (2003) On One Queuing System with Unreliable Queuing System. Problems of Applied Mechanics, 3, 9-18.

[7] Mikadze, I.S. and Khocholava, V.V. (2004) On One Model of Information Transition by Unreliable Communication Channel. Automatics and Telemechanics, 8, 85-90.

[8] Mikadze, I.S., Khocholava, V.V. and Khurodze, R.A. (2004) Virtual Waiting Time in Single-Line QS with Unreliable Devices. Automatics and Telemechanics, 12, 119-128.

[9] Khocholava, V.V. and Mikadze, I.S. (2002) On One Model of Queuing System. Georgian Engineering News, 4, 47-52. 
Scientific Research Publishing (SCIRP) is one of the largest Open Access journal publishers. It is currently publishing more than 200 open access, online, peer-reviewed journals covering a wide range of academic disciplines. SCIRP serves the worldwide academic communities and contributes to the progress and application of science with its publication.

Other selected journals from SCIRP are listed as below. Submit your manuscript to us via either submit@scirp.org or Online Submission Portal.
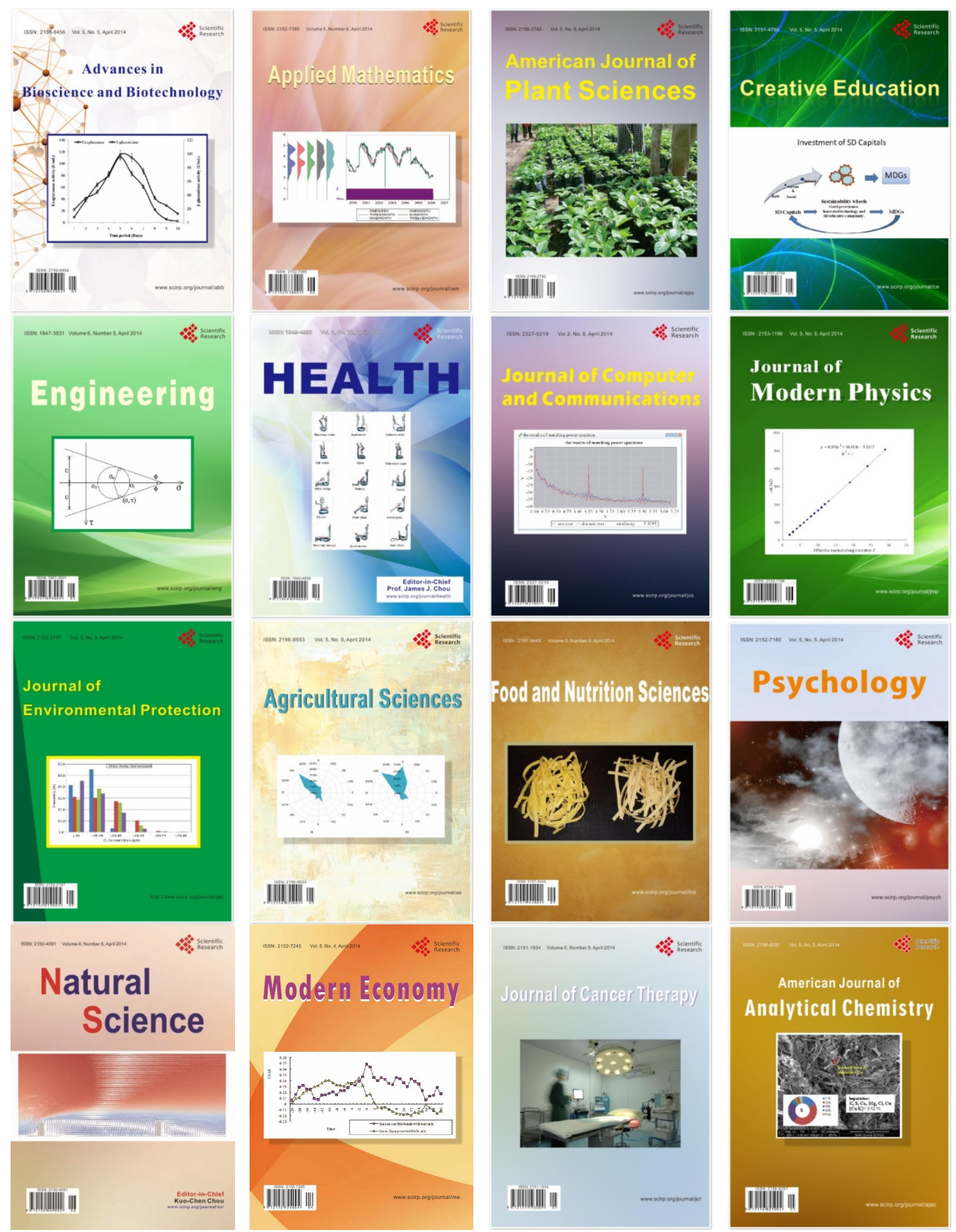